Acta Crystallographica Section B

\section{Structural}

Science

ISSN 0108-7681

\section{Gary S. Nichol and William Clegg*}

School of Natural Sciences - Chemistry, Bedson Building, University of Newcastle upon Tyne, Newcastle upon Tyne NE1 7RU, England

Correspondence e-mail: w.clegg@ncl.ac.uk

\title{
A variable-temperature study of a phase transition in barbituric acid dihydrate
}

The crystal structure of barbituric acid dihydrate $\left(\mathrm{C}_{4} \mathrm{H}_{4} \mathrm{~N}_{2} \mathrm{O}_{3} \cdot 2 \mathrm{H}_{2} \mathrm{O}\right)$ has twice been reported as orthorhombic, space group Pnma, with all atoms (except for $\mathrm{CH}_{2} \mathrm{H}$ atoms) lying on the mirror plane [Al-Karaghouli et al. (1977). Acta Cryst. B33, 1655-1660; Jeffrey et al. (1961). Acta Cryst. 14, 881-887]. The present study has found that at low temperatures, below $200 \mathrm{~K}$, the crystal structure is no longer orthorhombic but is non-merohedrally twinned monoclinic, space group $P 2_{1} / n$. This phase is stable down to $100 \mathrm{~K}$. Above $220 \mathrm{~K}$ the crystal structure is orthorhombic, and between 200 and $220 \mathrm{~K}$ the structure undergoes a phase change, with the monoclinic-to-orthorhombic phase transition itself taking place at around $216-217 \mathrm{~K}$. The size of the $\beta$ angle in the monoclinic structure is temperature dependent; at $100 \mathrm{~K} \beta$ is around $94^{\circ}$ and it decreases in magnitude towards $90^{\circ}$ as the temperature increases. Although the hydrogen-bonding motifs are the same for both crystal systems, there are significant differences in the crystal packing, in particular the out-of-plane displacement of the two water molecules and the $s p^{3}$-hybridized $\mathrm{C}$ atom of barbituric acid.

\section{Introduction}

Over the past few years, the topic of phase transitions has become more and more popular for scientific investigation. This extremely broad field is actively pursued by physicists, chemists, materials scientists, earth scientists and metallurgists (Pandey, 2005). Indeed, the January 2005 edition of Acta Crystallographica Section A: Foundations of Crystallography was devoted almost entirely to the topic. A simple search in February 2005 of SciFinder Scholar 2004 (American Chemical Society, 2004) for 'phase transition' resulted in almost 138500 hits; the top five years according to the greatest numbers of hits were 2003, 2002, 2001, 2000 and 2004. The number of hits in 2003 is 9013, more than double that of 1995 (4457) and a clear indication of the increasing interest in the subject.<smiles>O=C1C[Ge](=O)NC(=O)N1</smiles>

Barbituric acid dihydrate (I) with atomic numbering scheme
(C) 2005 International Union of Crystallography Printed in Great Britain - all rights reserved
Received 5 March 2005

Accepted 31 May 2005 
parent molecule of the barbiturate family of drugs, which are of crystallographic interest not least for their propensity to form polymorphs. The 5,5-dialkyl derivatives are those which are pharmacologically active and which have been most extensively characterized by X-ray crystallography (Caillet \& Claverie, 1980; Cleverley \& Williams, 1959; Craven et al., 1969, 1982; Craven \& Vizzini, 1969, 1971; McMullan et al., 1978; Nichol \& Clegg, 2005a,b; Platteau et al., 2005; Sambyal et al., 1995; Williams, 1973, 1974). Contemporary research continues to focus on barbituric acid polymorphism as a model system for developing computational polymorph prediction techniques, something that is of major importance to the pharmaceutical industry (Lewis et al., 2004, 2005).

\subsection{Analysis of current literature}

The structure of barbituric acid dihydrate (I) appears twice in the primary literature: an X-ray diffraction study (Jeffrey et al., 1961) and a neutron diffraction study (Al-Karaghouli et al., 1977). In both reports the data collections were carried out at room temperature, and the crystal system and space group are reported as orthorhombic, Pnma. The final $R$ factors are 0.14 and 0.087 , respectively. Both reports conclude that, with the exception of the two $\mathrm{H}$ atoms of the $\mathrm{CH}_{2}$ group, all atoms of the barbituric acid and water molecules lie on the mirror plane. During their discussions, both reports make mention of the high atomic displacement observed in the $b$-axis direction (i.e. perpendicular to the mirror plane). Al-Karaghouli et al. (1977) considered the possibility of an alternative noncentrosymmetric space group, $P n 2_{1} a$ (non-standard setting of $P n a 2_{1}$ ), which would allow the atoms to deviate from the (now non-crystallographic) mirror plane. These authors also considered a model in which one of the $\mathrm{O}$ atoms was deliberately displaced off the mirror plane and then refined as disordered. Neither of these models gave a satisfactory outcome and they concluded that there was no good reason to doubt the assignment of Pnma as the space group.

\section{Experimental}

\subsection{Preliminary experiments}

With these uncertainties in mind, we carried out a lowtemperature redetermination of barbituric acid dihydrate for the purpose of having a reference structure of the ligand for reliable comparison with the structures of our metal complexes, also determined routinely at low temperature. It was found that, at $150 \mathrm{~K}$, the crystal system was not orthorhombic but non-merohedrally twinned monoclinic and the space group was $P 2_{1} / n$. Curious to know whether this result pointed to inaccuracies in the literature reports (which were at least 27 years old), we re-collected data, from the same crystal, at room temperature. As reported by Jeffrey et al. (1961), the crystal decomposed on the diffractometer during data collection from a transparent colourless crystal to a white opaque solid, which did not diffract at all. Nevertheless, sufficient data were collected to confirm that at room temperature the structure is indeed orthorhombic with the space group Pnma.
Hence the crystal had undergone a phase transition on warming from low temperature to room temperature (and, presumably, in the reverse direction in the initial flashcooling). A variable-temperature X-ray diffraction study was carried out to observe the effect of changing temperature on the crystal structure and to determine at what point the phase transition occurs.

\subsection{Sample preparation}

Crystals of barbituric acid dihydrate were prepared by dissolving a sample of commercially available barbituric acid (obtained as a white powder from Lancaster Synthesis) in distilled water with gentle heating. Storage at $278 \mathrm{~K}$ over a weekend resulted in large colourless and perfectly transparent block crystals of barbituric acid dihydrate.

\subsection{Experimental strategy}

Data were collected on a Bruker SMART $1 \mathrm{~K}$ CCD diffractometer fitted with an Oxford Cryosystems Cryostream cooler (Cosier \& Glazer, 1986) at 14 different temperatures ranging from 100 to $270 \mathrm{~K}$. Experimental details for selected temperatures are summarized in Table 1 (details for all experiments are available in the deposited supplementary material ${ }^{\mathbf{1}}$ ). A large good-quality crystal, which did not require cutting, was selected from the sample and, on the basis of preliminary experiments, the experimental strategy was started by re-collecting data at $150 \mathrm{~K}$ and then proceeding in the following temperature order: 170, 190, 200, 210, 220, 230, $215,217,218,219,216,100$ and $270 \mathrm{~K}$. A full data collection, as opposed to a simple unit-cell determination, was carried out at each temperature. Such a procedure adds several days to the time taken to conduct the experiments; however, it also allows for complete structure solution and refinement - the ultimate indicator of crystal system correctness and data quality - at each temperature and is especially important when one considers that the crystals were twinned in the monoclinic crystal system; a full data collection allows the determination of unit-cell parameters for both components of the twin from several hundred reflections, rather than the hundred or so that would be measured by only collecting partial data for an orientation matrix. The same data collection strategy (complete sphere of reciprocal space, $0.3^{\circ}$ width frames, $30 \mathrm{~s}$ exposures) was used for each experiment.

The reasons for selecting two extreme temperatures to finish the strategy were to check that the crystal did not undergo a second phase transition at even lower temperatures; so we could verify that the phase transition is reversible; so that we could see that the crystal did not suffer physical stress at extreme cold; and so we could collect data as close to room temperature as possible without the crystal decomposing. The same crystal, pictured in Fig. 1, was used for every experiment;

\footnotetext{
${ }^{1}$ Supplementary data for this paper are available from the IUCr electronic archives (Reference: WS5026). Services for accessing these data are described at the back of the journal.
} 
Table 1

Experimental details at selected temperatures.

Details for all experiments are given in the deposited CIF.

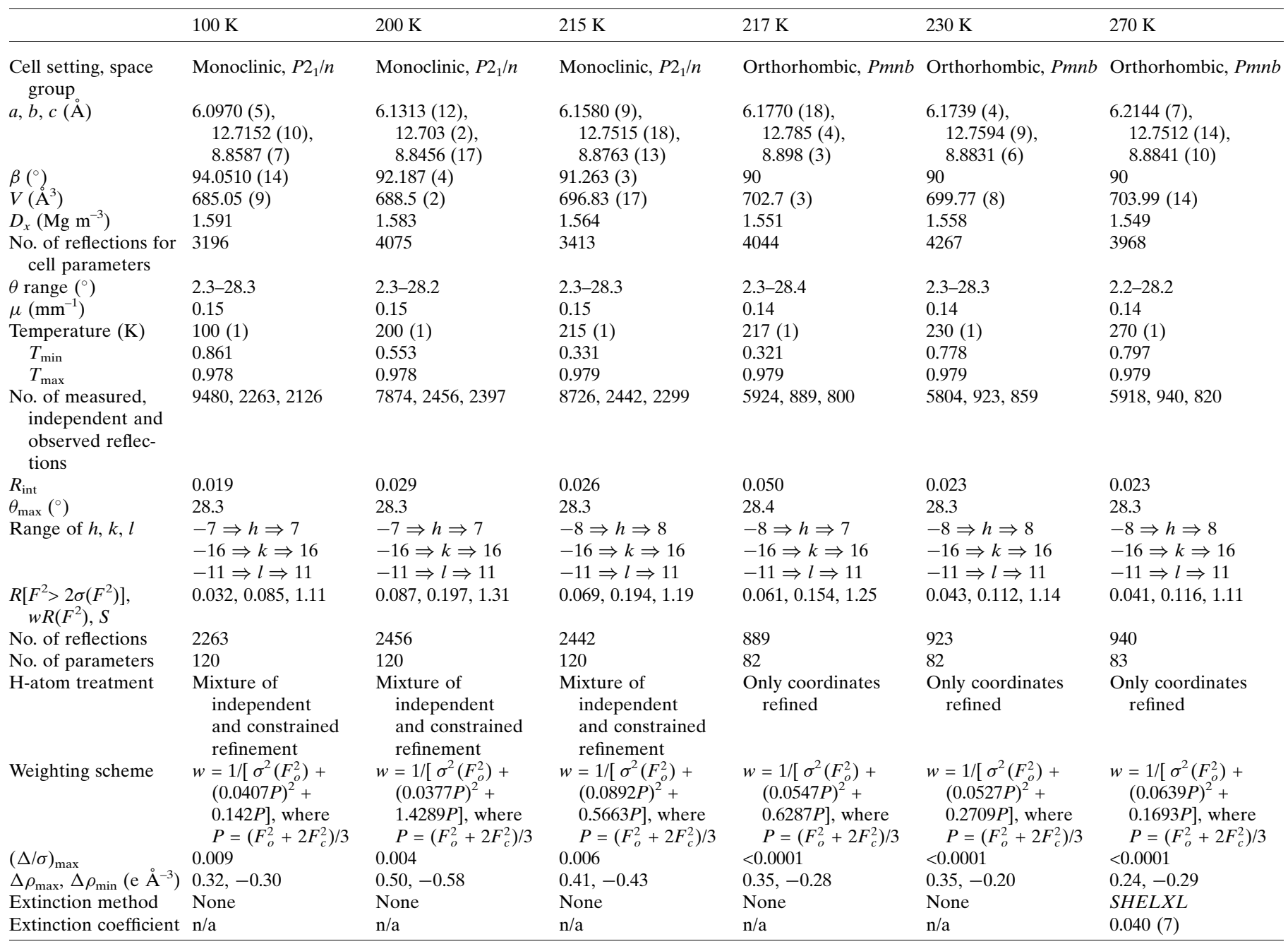

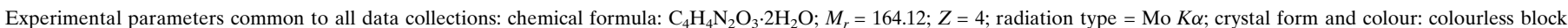

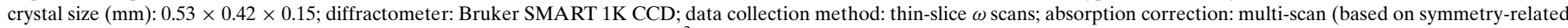

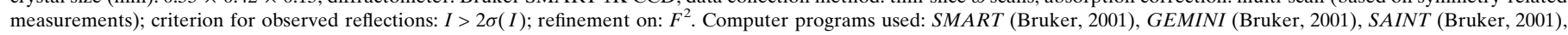
SHELXTL (Sheldrick, 2001) and local programs.

the crystal was not removed from the goniometer head between data collections, and a visual examination of the crystal at the end of the experiments showed that it suffered no physical effects (e.g. cracking) as a result of the cooling and heating. Ultimately the same crystal stayed attached to the goniometer head for over 2 weeks.

The true crystal temperature was verified by collecting data on a crystal of $\mathrm{CsOH} \cdot \mathrm{H}_{2} \mathrm{O}$ (purchased from Lancaster Synthesis). Caesium hydroxide monohydrate is known to undergo a phase transition from $C$-centred monoclinic to hexagonal at $229 \mathrm{~K}$ (Tomaszewski, 1992). This phase transition was observed at $228-229 \mathrm{~K}$ and so the crystal temperature as reported by the Cryostream was found to be reliable. After each temperature change the crystal of barbituric acid dihydrate was allowed to stabilize at the new temperature for around $30 \mathrm{~min}$ before starting the data collection.

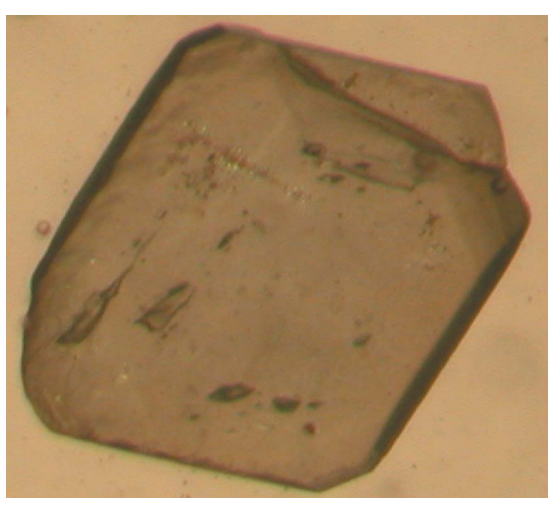

Figure 1

The crystal after two weeks on the diffractometer. The apparent defect at the top right is a ridge in the crystal and not a crack. Other apparent defects on the face of the crystal are air bubbles in the oil used to coat and store the crystal. 
Table 2

Summary of results for refinements at each temperature.

\begin{tabular}{|c|c|c|c|c|c|}
\hline \multirow[b]{2}{*}{$\begin{array}{l}\text { Temperature } \\
(\mathrm{K})\end{array}$} & \multirow[b]{2}{*}{$\begin{array}{l}\text { Space } \\
\text { group }\end{array}$} & \multicolumn{2}{|c|}{ Data to $2 \theta=52^{\circ}$} & \multicolumn{2}{|c|}{ Data to $2 \theta=50^{\circ}$} \\
\hline & & $\begin{array}{l}R \\
{\left[F_{o}^{2}>4 \sigma\left(F_{o}^{2}\right)\right]}\end{array}$ & $\begin{array}{l}w R \\
\left(\text { all } F^{2}\right)\end{array}$ & $\begin{array}{l}R \\
{\left[F_{o}^{2}>4 \sigma\left(F_{o}^{2}\right)\right]}\end{array}$ & $\begin{array}{l}w R \\
\left(\text { all } F^{2}\right)\end{array}$ \\
\hline 100 & $P 2_{1} / n$ & 0.0319 & 0.0827 & 0.0291 & 0.0927 \\
\hline 150 & $P 2_{1} / n$ & 0.0505 & 0.1309 & 0.0384 & 0.1098 \\
\hline 170 & $P 2_{1} / n$ & 0.0397 & 0.1027 & 0.0341 & 0.1000 \\
\hline 190 & $P 2_{1} / n$ & 0.0374 & 0.1004 & 0.0343 & 0.1124 \\
\hline 200 & $P 2_{1} / n$ & 0.0869 & 0.1967 & 0.0618 & 0.1540 \\
\hline 210 & $P 2_{1} / n$ & 0.0664 & 0.1496 & 0.0462 & 0.1176 \\
\hline 215 & $P 2_{1} / n$ & 0.0690 & 0.1919 & 0.0522 & 0.1591 \\
\hline 216 & $P 2_{1} / n$ & 0.0684 & 0.1806 & 0.0508 & 0.1477 \\
\hline 217 & Pmnb & 0.0611 & 0.1504 & 0.0469 & 0.1277 \\
\hline 218 & Pmnb & 0.0539 & 0.1337 & 0.0425 & 0.1156 \\
\hline 219 & Pmnb & 0.0664 & 0.1597 & 0.0479 & 0.1264 \\
\hline 220 & Pmnb & 0.0453 & 0.1198 & 0.0391 & 0.1083 \\
\hline 230 & Pmnb & 0.0429 & 0.1095 & 0.0332 & 0.0914 \\
\hline 270 & Pmnb & 0.0415 & 0.1239 & 0.0323 & 0.0886 \\
\hline
\end{tabular}

\subsection{Data processing}

For each collection the data were processed both as monoclinic and as orthorhombic, regardless of the symmetry implied by the data. This approach proved especially important for those data sets collected around the transition temperature. For example, those data sets which were clearly monoclinic were also processed as orthorhombic, with the $\beta$ angle constrained in cell refinement after integration and the space group set as Pmnb. We chose this unconventional setting of Pnma so that the unit-cell axes matched those of the monoclinic space group $P 2_{1} / n$, thus allowing for detailed comparison of the two structures. Similarly the orthorhombic data sets were integrated as monoclinic with no constraints on the $\beta$ angle and the space group $P 2_{1} / n$ selected. By treating each data set in this way and comparing the final monoclinic and orthorhombic refinement results it was, in most cases, obvious which was correct and which was incorrect.

Starting with the $150 \mathrm{~K}$ collection the programs GEMINI and $S M A R T$ (Bruker, 2001) were used to determine and refine both components of the twin. SAINT (Bruker, 2001) was then used to integrate the data and TWINABS (Sheldrick, 2002) was used to correct for absorption and other effects and to write two corrected data files for structure solution and refinement. The SHELXTL suite of programs was used for space group determination, structure solution and refinement (Sheldrick, 2001). Having refined the structure as non-merohedrally twinned monoclinic to a satisfactory conclusion the data processing was repeated as described above with orthorhombic constraints. We used $S A D A B S$ (Sheldrick, 2003) and not TWINABS for absorption correction of the (untwinned) orthorhombic data sets. Molecular diagrams and other graphics were produced using DIAMOND (Brandenburg \& Putz, 2004) and MERCURY (Version 1.3; Bruno et al., 2002).

This approach was followed for all other data collections, and the non- $\mathrm{H}$ atomic coordinates from the $150 \mathrm{~K}$ collection were used as starting parameters for structure refinement at all other temperatures. This procedure ensured that factors such as unit-cell origin, atomic coordinates and atomic labels were consistent throughout. Appropriate adjustments were made to the coordinates of the structures in Pmnb to constrain the atoms to lie on the mirror plane in accordance with the spacegroup symmetry. Anomalies in some of the transmission factor ranges are discussed below.

\section{Results and discussion}

A summary of the refinement results for each data collection is presented as Table 2. Examination of the results at each temperature shows that it is possible to classify each one as definitely monoclinic, definitely orthorhombic or 'transitional', where it is not immediately obvious which is the most appropriate space group, and in some cases both crystal systems seem appropriate. The ADDSYM function of PLATON (Spek, 2003) was very useful in the detection of additional symmetry in the monoclinic structures.

\subsection{Diffraction patterns}

Examination of the diffraction pattern is the most reliable way of determining the correct crystal system of a structure. As a simple example, Fig. 2 shows three screenshots of a frame recorded at 100,215 and $230 \mathrm{~K}$ with the crystal in the same orientation. On each frame two pairs of reflections have been highlighted. They share common $h$ and $k$ indices but differ in the value of $l$ (as indicated on the $230 \mathrm{~K}$ frame). One reflection of each pair belongs to one component of the twin and the other reflection belongs to the second component of the twin. The two components are related by a $180^{\circ}$ rotation about the $c$ axis. At $100 \mathrm{~K}$, a monoclinic temperature, the reflections are well separated and the program GEMINI could easily index both twin components. As the temperature increases the reflections begin to move closer together and at $215 \mathrm{~K}$, a transitional temperature, they are starting to merge. Indexing the diffraction pattern is now not so easy, and both monoclinic and orthorhombic unit cells can be determined. At $230 \mathrm{~K}$ pairs of reflections have merged completely, to give discrete reflections with unique indices, and the structure is now orthorhombic.

\subsection{Unit-cell parameters}

Table 3 gives unit-cell parameters for all experiments. Phase transitions are often accompanied by a significant change in unit-cell dimensions, such as the doubling of an axis. Here there is little change in the size of the unit cell save for a gradual increase in unit-cell volume so that the unit cell at $270 \mathrm{~K}$ is around $19 \AA^{3}$ larger than that at $100 \mathrm{~K}$. This difference is largely insignificant, given that unit cells measured at or near room temperature are generally larger than those measured at low temperatures.

\subsection{Orthorhombic structures}

Data collected at 220, 230 and $270 \mathrm{~K}$ are classed as definitely orthorhombic. At these temperatures GEMINI was unable to determine two separate twin components from the 
Table 3

Unit-cell parameters for all data collections.

\begin{tabular}{llllllll}
\hline $\begin{array}{l}\text { Tempera- } \\
\text { ture (K) }\end{array}$ & $a$ & $b$ & $c$ & $\alpha$ & $\beta$ & $\gamma$ & Volume \\
\hline 100 & $6.0970(5)$ & $12.7152(1)$ & $8.8587(7)$ & 90 & $94.051(1)$ & 90 & $685.05(9)$ \\
150 & $6.1130(8)$ & $12.7149(2)$ & $8.8564(1)$ & 90 & $93.437(2)$ & 90 & $687.14(2)$ \\
170 & $6.1270(5)$ & $12.7253(1)$ & $8.8633(8)$ & 90 & $93.068(2)$ & 90 & $690.06(1)$ \\
190 & $6.1377(5)$ & $12.7306(1)$ & $8.8641(8)$ & 90 & $92.528(2)$ & 90 & $691.94(1)$ \\
200 & $6.1313(1)$ & $12.7032(2)$ & $8.8456(2)$ & 90 & $92.187(4)$ & 90 & $688.45(2)$ \\
210 & $6.1538(2)$ & $12.7474(3)$ & $8.8776(2)$ & 90 & $91.627(4)$ & 90 & $696.05(3)$ \\
215 & $6.1580(9)$ & $12.7515(2)$ & $8.8963(1)$ & 90 & $91.263(3)$ & 90 & $698.40(2)$ \\
216 & $6.1567(2)$ & $12.7329(3)$ & $8.8646(2)$ & 90 & $91.180(5)$ & 90 & $694.77(3)$ \\
217 & $6.1770(2)$ & $12.7851(2)$ & $8.8984(3)$ & 90 & 90 & 90 & $702.70(3)$ \\
218 & $6.1626(2)$ & $12.7574(4)$ & $8.8763(1)$ & 90 & 90 & 90 & $697.80(4)$ \\
219 & $6.1624(2)$ & $12.7569(3)$ & $8.8782(2)$ & 90 & 90 & 90 & $697.94(3)$ \\
220 & $6.1665(1)$ & $12.7626(4)$ & $8.8814(2)$ & 90 & 90 & 90 & $698.99(2)$ \\
230 & $6.1739(4)$ & $12.7594(9)$ & $8.8831(6)$ & 90 & 90 & 90 & $699.77(8)$ \\
270 & $6.2144(7)$ & $12.7512(1)$ & $8.8841(1)$ & 90 & 90 & 90 & $703.99(1)$ \\
\hline
\end{tabular}

diffraction patterns so the possibility that the data were nonmerohedrally twinned was discarded. Orthorhombic and pseudo-orthorhombic models both gave similar satisfactory values of $R$ when refinement had converged, so we examined

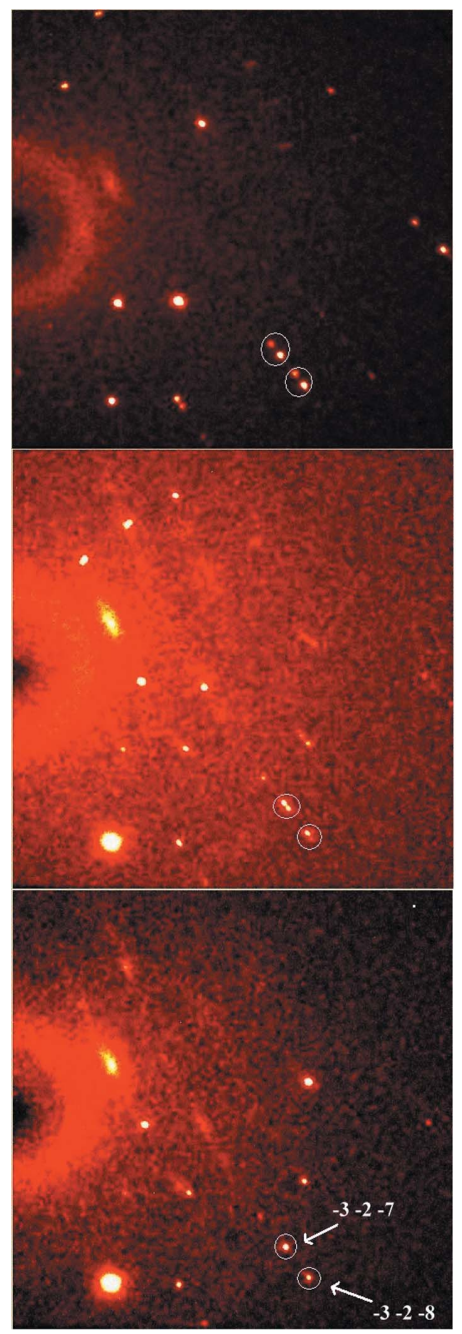

Figure 2

Three frames from data collections at $100 \mathrm{~K}$ (top), $215 \mathrm{~K}$ (centre) and $230 \mathrm{~K}$ (bottom). the pseudo-orthorhombic models for additional symmetry. The use of ROTAX (Cooper et al., 2002) showed that $180^{\circ}$ rotations were possible about the [100], [010] and [001] reciprocal and direct lattice directions, and analysis with $A D D S Y M$ showed an additional mirror plane missing from the model. It was simple to conclude that, at these temperatures, the structures are indeed, as has twice been reported, best described in the higher-symmetry space group Pmnb (or Pnma) rather than in $P 2_{1} / n$.

Taking the structure at $230 \mathrm{~K}$ as an example, a displacement ellipsoid plot and a packing diagram viewed along the $c$ axis of (I) are given in Fig. 3. $\mathrm{H}$ atoms were all located in a difference map and refined with $U_{\text {iso }}=1.2 U_{\text {eq }}(\mathrm{C}, \mathrm{N}, \mathrm{O})$; their coordinates were refined freely. All atoms, with the exception of the $\mathrm{CH}_{2} \mathrm{H}$ atoms, lie on the mirror plane (one of the $\mathrm{H}$ atoms in the ellipsoid plot is symmetry generated); this fact is neatly shown by the packing diagram. The two water molecules are coplanar with the barbituric acid ring. Molecular dimensions are unexceptional and in agreement with those reported by AlKaraghouli et al. (1977), with the exception of the $X-\mathrm{H}$ bonds, which are around $0.1-0.2 \AA$ shorter than the previously reported values. This difference is to be expected, since ours is
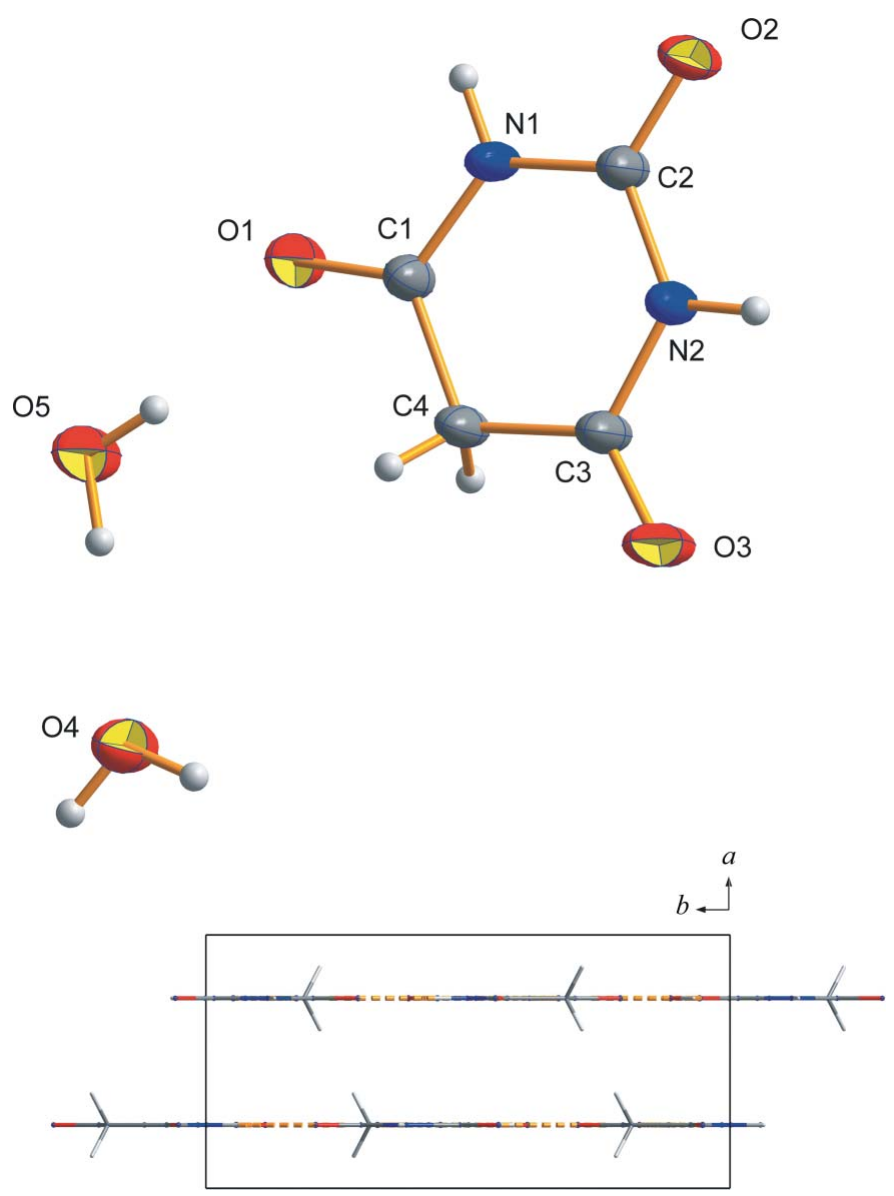

Figure 3

Displacement ellipsoid plot (50\% probability) and packing diagram along the $c$ axis of the $230 \mathrm{~K}$ structure. Hydrogen bonds are indicated in orange. 
Table 4

Selected geometric parameters $\left(\AA,^{\circ}\right)$ from the monoclinic $100 \mathrm{~K}$ structure.

\begin{tabular}{lllllr}
\hline $\begin{array}{l}\text { Atom } \\
\text { sites }\end{array}$ & $\begin{array}{l}\text { Bond } \\
\text { lengths }\end{array}$ & Atom sites & $\begin{array}{l}\text { Bond } \\
\text { angles }\end{array}$ & Atom sites & \multicolumn{1}{l}{$\begin{array}{l}\text { Torsion } \\
\text { angles }\end{array}$} \\
\hline $\mathrm{N} 1-\mathrm{C} 1$ & $1.3643(13)$ & $\mathrm{O} 1-\mathrm{C} 1-\mathrm{C} 4$ & $121.62(9)$ & $\mathrm{C} 2-\mathrm{N} 1-\mathrm{C} 1-\mathrm{C} 4$ & $-4.70(16)$ \\
$\mathrm{N} 1-\mathrm{C} 2$ & $1.3810(13)$ & $\mathrm{N} 1-\mathrm{C} 1-\mathrm{C} 4$ & $117.59(9)$ & $\mathrm{C} 2-\mathrm{N} 2-\mathrm{C} 3-\mathrm{C} 4$ & $6.22(17)$ \\
$\mathrm{N} 2-\mathrm{C} 2$ & $1.3728(13)$ & $\mathrm{O} 3-\mathrm{C} 3-\mathrm{C} 4$ & $123.01(9)$ & $\mathrm{O} 1-\mathrm{C} 1-\mathrm{C} 4-\mathrm{C} 3$ & $-172.47(10)$ \\
$\mathrm{N} 2-\mathrm{C} 3$ & $1.3670(13)$ & $\mathrm{N} 2-\mathrm{C} 3-\mathrm{C} 4$ & $117.29(9)$ & $\mathrm{N} 1-\mathrm{C} 1-\mathrm{C} 4-\mathrm{C} 3$ & $9.09(15)$ \\
$\mathrm{C} 1-\mathrm{C} 4$ & $1.5034(14)$ & $\mathrm{C} 1-\mathrm{C} 4-\mathrm{C} 3$ & $115.90(8)$ & $\mathrm{O} 3-\mathrm{C} 3-\mathrm{C} 4-\mathrm{C} 1$ & $171.19(11)$ \\
$\mathrm{C} 3-\mathrm{C} 4$ & $1.5054(14)$ & & & $\mathrm{N} 2-\mathrm{C} 3-\mathrm{C} 4-\mathrm{C} 1$ & $-9.76(15)$ \\
\hline
\end{tabular}

towards the phase transition. All of these structures share another common feature in that the barbituric acid molecule is no longer planar. In this space group there is no imposed mirror symmetry and as a result the $\mathrm{Csp}^{3}$ (C4) atom, with its tetrahedral rather than trigonal geometry, is seen to deviate from the mean plane of the rest of the molecule.

Fig. 4 shows a displacement ellipsoid plot of (I) at $100 \mathrm{~K}$. All $\mathrm{H}$ atoms were identified in a difference electron density map and their coordinates were refined, with the exception of the $\mathrm{CH}_{2} \mathrm{H}$ atoms, which were positioned geometrically $(\mathrm{C}-\mathrm{H}=0.99 \AA)$ and

an X-ray diffraction study and we are comparing it with neutron diffraction results.

\subsection{Monoclinic structures}

Those structures determined at 100, 150, 170 and $190 \mathrm{~K}$ are classed as definitely monoclinic with space group $P 2_{1} / n$. In each case the diffraction pattern is non-merohedrally twinned. That the diffraction pattern is twinned as a result of the orthorhombic-to-monoclinic transition is not surprising and is quite common in situations of a material changing from higher to lower symmetry. The two components of the twin are related by a $180^{\circ}$ rotation about the $c$ axis, and at low temperatures the extent of the twinning is such that one can clearly see the reflections from both components in the diffraction pattern, as shown in Fig. 2. Attempts to refine these data with orthorhombic models result in refinements with very large $R$ factors. Another curious feature is the change in the magnitude of the $\beta$ angle with temperature; as shown in Table 3 , the $\beta$ angle approaches $90^{\circ}$ as the temperature increases
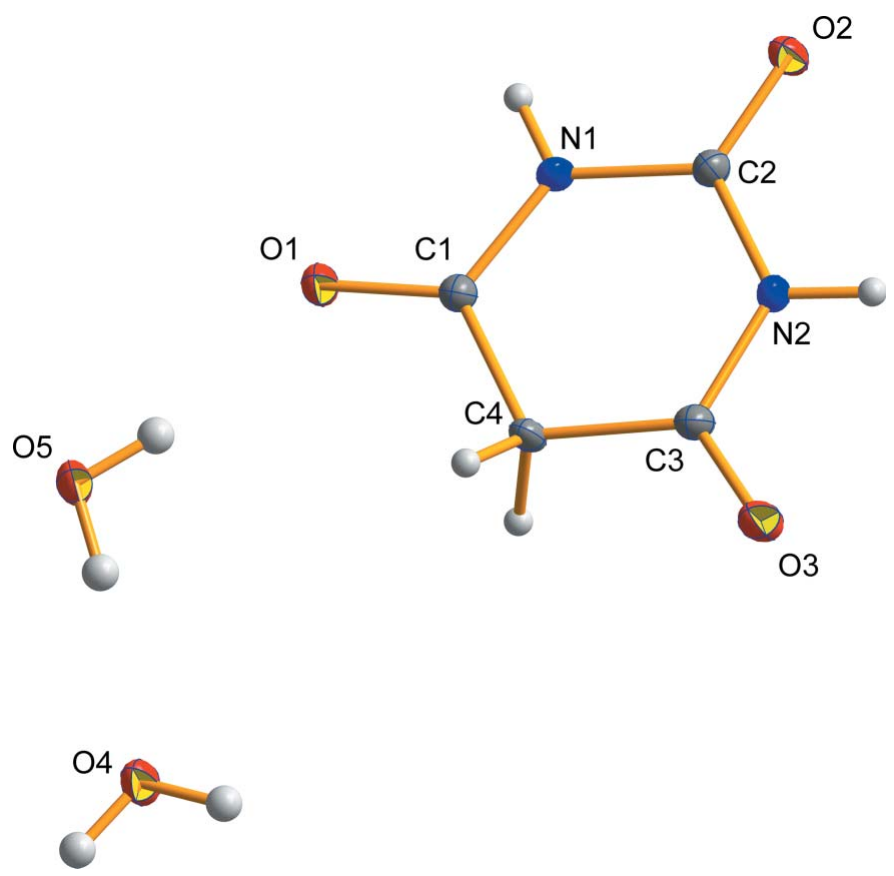

Figure 4

Displacement ellipsoid plot (50\% probability) of the $100 \mathrm{~K}$ structure. constrained as riding during refinement. All $\mathrm{H}$ atoms were refined with $U_{\text {iso }}=1.2 U_{\text {eq }}(\mathrm{O}, \mathrm{N}, \mathrm{C})$. Molecular dimensions, listed in Table 4, are unexceptional and, with the exception of the torsion angles, are more or less the same as those determined at $230 \mathrm{~K}$. Fig. 5 shows an overlay of the monoclinic structure at $100 \mathrm{~K}$ (red) and the orthorhombic structure at $230 \mathrm{~K}$ (black), produced by plotting the mean plane (r.m.s. deviation $0.0288 \AA$ ) of atoms $\mathrm{C} 1, \mathrm{O} 1, \mathrm{~N} 1, \mathrm{C} 2, \mathrm{O} 2, \mathrm{C} 2, \mathrm{~N} 2, \mathrm{C} 3$ and $\mathrm{O} 3$ of the monoclinic $100 \mathrm{~K}$ structure against the planar ring of the orthorhombic $230 \mathrm{~K}$ structure. The out-of-plane displacement of the $\mathrm{C} 4$ atom can be clearly seen. This is not an unprecedented observation; the structure of unsolvated barbituric acid shows a similar puckering in the ring (Bolton, 1963; Lewis et al., 2004). By using the CALCALL function of $P L A T O N$ we determined the Cremer-Pople ring puckering parameter $Q$ at $100 \mathrm{~K}$ to be $0.0787 \AA$. This is a very small value but does indicate that at $100 \mathrm{~K}$ the ring is distorted to a measurable degree in the envelope conformation. At higher temperatures the ring puckering is less significant and CALCALL does not report it. This small, but significant, conformational flexibility of the barbituric acid molecule has proved to be a major obstacle in polymorph prediction (Lewis et al., 2004).

In addition to the ring puckering, the two water molecules are no longer coplanar with the barbituric acid ring. This is a more significant change from the orthorhombic structure and, as a consequence, the molecular packing shows some obvious differences. Fig. 6 shows a packing diagram of the structure at $100 \mathrm{~K}$, viewed along the $c$ axis. The hydrogen-bonding motifs in both the orthorhombic and the monoclinic structures are identical but here, because the water molecules are no longer coplanar with the barbituric acid molecules, some adjustment in the packing is necessary to preserve the hydrogen-bonding arrangement. Thus, instead of observing perfectly planar sheets of hydrogen-bonded water and barbituric acid molecules, we see sheets that are now rippled in appearance. Hydrogen-bonding parameters are given in Table 5.

\subsection{Transitional structures}

The structures refined from data collected between 200 and $219 \mathrm{~K}$ are classed as 'transitional'; that is to say, aspects of the data and the refinement imply that the structure is undergoing change of some sort. Table 6 gives details of the final refinement outcomes for both space groups and shows also the 
Table 5

Hydrogen-bonding geometry in the monoclinic $100 \mathrm{~K}$ structure.

\begin{tabular}{lllll}
\hline & $D-\mathrm{H}$ & $\mathrm{H} \cdots A$ & $D \cdots A$ & $D-\mathrm{H} \cdots A$ \\
\hline $\mathrm{O} 4-\mathrm{H} 1 \mathrm{O} \cdots \mathrm{O} 2^{\mathrm{i}}$ & $0.819(17)$ & $1.969(17)$ & $2.7583(11)$ & $161.9(16)$ \\
$\mathrm{O} 4-\mathrm{H} 2 \mathrm{O} \cdots \mathrm{O} 1^{\text {ii }}$ & $0.822(17)$ & $2.034(17)$ & $2.8508(11)$ & $172.4(15)$ \\
$\mathrm{O} 5-\mathrm{H} 3 \mathrm{O} \cdots \mathrm{O} 4$ & $0.821(18)$ & $1.931(19)$ & $2.7463(12)$ & $171.7(17)$ \\
$\mathrm{O} 5-\mathrm{H} 4 \mathrm{O} \cdots \mathrm{O} 1$ & $0.828(17)$ & $1.967(18)$ & $2.7819(12)$ & $167.9(16)$ \\
$\mathrm{N} 1-\mathrm{H} 1 \mathrm{~N} \cdots 3^{\text {iii }}$ & $0.823(15)$ & $1.986(16)$ & $2.8084(12)$ & $177.2(14)$ \\
$\mathrm{N} 2-\mathrm{H} 2 \mathrm{~N} \cdots 5^{\text {iv }}$ & $0.874(15)$ & $1.861(15)$ & $2.7277(12)$ & $171.2(14)$ \\
\hline
\end{tabular}

Symmetry codes: (i) $\frac{1}{2}-x, \frac{1}{2}+y, \frac{3}{2}-z$; (ii) $\frac{1}{2}-x, \frac{1}{2}+y, \frac{1}{2}-z$; (iii) $\frac{1}{2}-x,-\frac{1}{2}+y, \frac{3}{2}-z$; (iv) $x$, $y, 1+z$.

unconstrained $\beta$ angle as determined in the monoclinic models. At these temperatures the choice of monoclinic versus orthorhombic was not immediately obvious, and several different approaches to each data set were tried in order to determine which cell setting and space group best described the data.

As can be seen from the refinement results presented in Tables 2 and 6, data quality at these temperatures was much poorer than those at lower or higher temperatures. In particular, the data above $2 \theta=50^{\circ}$ were much weaker than previously observed, and removal of these data from the refinement led to a marked improvement in the quality of $R$ and $w R$. High-angle data quality usually depends on factors such as crystal size and quality, scattering power of the atoms,
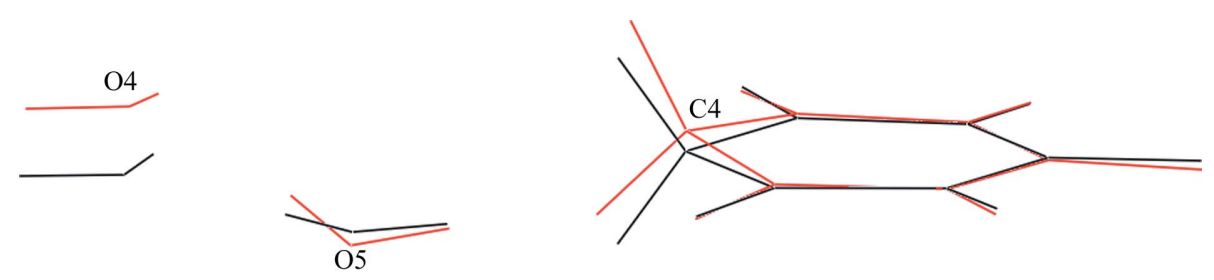

Figure 5

Overlay of the $100 \mathrm{~K}$ structure (red) and $230 \mathrm{~K}$ structure (black) showing the out-of-plane displacement of the $\mathrm{C} 4$ atom and the two water molecules.

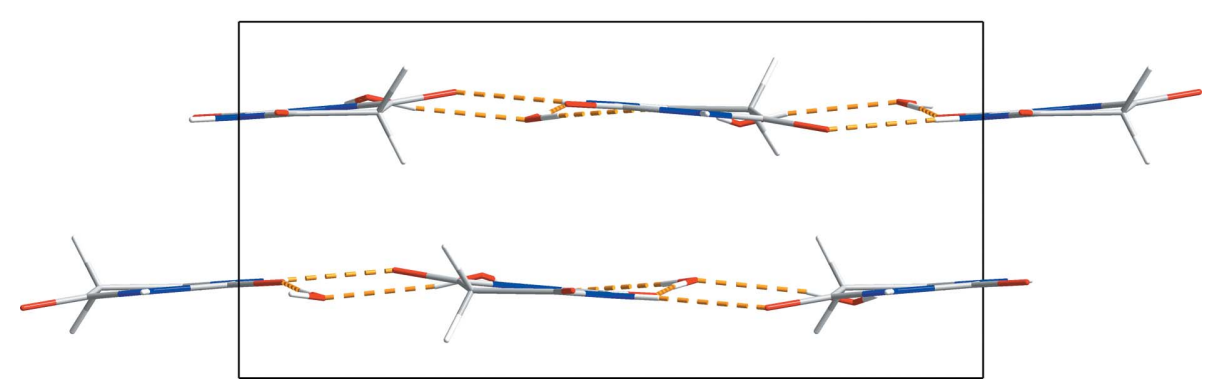

Figure 6

Packing diagram along the $c$ axis of the $100 \mathrm{~K}$ structure. Hydrogen bonds are marked in orange.

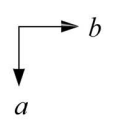

disorder within the structure, and temperature of data collection. In this study the same crystal was used throughout and the structure is rigid with little scope for disorder, leaving just the effect of increasing temperature as a possible cause of weaker high-angle data. It is true that the lower the crystal temperature, the higher the diffracted X-ray intensities are, and so the more distinguishable from the background are the reflections. However, this fact would not account for such a marked decrease in the data quality from 190 to $200 \mathrm{~K}$. Usually in such a case one would be justified in omitting these poor data from the least-squares calculations. However, this approach would not be appropriate in this case. That the highangle data at transitional temperatures are poor in comparison to other collections is a significant observation in this study, and it is for this reason that the resolution of the refinement and structure reporting were not restricted to $2 \theta_{\max }=50^{\circ}$.

Another significant observation is the difference between the minimum and maximum transmission factors resulting from the TWINABS/SADABS scaling, as presented in Table 1. The differences between $T_{\min }$ and $T_{\max }$ at 100,230 and $270 \mathrm{~K}$ are reasonable; however, those reported at 200, 215 and $217 \mathrm{~K}$ are not. TWINABS and $S A D A B S$ correct for absorption by comparing the intensities of supposedly equivalent (by symmetry) or repeated (as a result of collecting redundant data) reflections. Given that the same crystal was used for all experiments, the large range of transmission at these intermediate temperatures cannot be connected to the shape or size of the crystal. Each data set was collected using an identical strategy, ruling out the possibility of variation due to changes in experimental settings. The wide variations in the putative absorption corrections must be a partial compensation for the poor quality of data from an intermediate structural state by the framescaling procedure in these multi-scan correction methods. We do not believe the variations are due to any hysteresis effect of structural change lagging behind temperature change, since the phase transition is not a large one, and the crystal was held at each new temperature for at least $30 \mathrm{~min}$ before data collection began. However, it should be noted that the temperature interval between these data sets is of the same order as the uncertainty in the temperature itself. If the phase transition is one that takes place gradually over a range of several degrees in temperature then some minor variation in the structure, and hence in the diffraction pattern, during each data collection is likely. These intermediate structures should each be regarded as an average 
Table 6

Comparison of refinement details for transitional structures.

\begin{tabular}{|c|c|c|c|c|c|c|}
\hline \multirow{2}{*}{$\begin{array}{l}\text { Temperature } \\
(\mathrm{K})\end{array}$} & \multirow{2}{*}{$\begin{array}{l}\beta \text { angle } \\
\text { (unconstrained) }\end{array}$} & \multicolumn{2}{|c|}{$R$ for $2 \theta<52^{\circ}$} & \multicolumn{2}{|c|}{$R$ for $2 \theta<50^{\circ}$} & \multirow{2}{*}{$\begin{array}{c}\text { Mirror plane detected by } \\
\qquad A D D S Y M \text { (in } P 2_{1} / n \text { ? }\end{array}$} \\
\hline & & $P 2_{1} / n$ & Pnmb & $P 2_{1} / n$ & Pnmb & \\
\hline 200 & 92.187 (4) & 0.0869 & 0.1020 & 0.0618 & 0.0669 & No \\
\hline 215 & $91.263(3)$ & 0.0690 & 0.0865 & 0.0522 & 0.0632 & No \\
\hline 216 & $91.180(5)$ & 0.0684 & 0.0743 & 0.0508 & 0.0546 & No \\
\hline 217 & $90.952(4)$ & 0.0741 & 0.0611 & 0.0596 & 0.0469 & Yes \\
\hline 218 & 90.139 (4) & 0.0670 & 0.0539 & 0.0500 & 0.0425 & Yes \\
\hline
\end{tabular}

structure over a small temperature range, and the range of transmission factors probably reflects this, together with the generally poorer refinement results compared with those at higher and lower temperatures where a single phase is present.

3.5.1. Structures at 200, 210, 215 and $216 \mathrm{~K}$. After much experimentation, several unit-cell determinations, the creation of many different models and seemingly endless refinement cycles, it was concluded that, at these temperatures, the crystal structures are better described as monoclinic rather than orthorhombic. However, in each case the decision was very close and, if taken based on refinement alone, would have been difficult to determine. To verify that orthorhombic was not a more appropriate description of the data, $A D D S Y M$ was used to detect missed symmetry and in each case none was detected. The data collected at 215 and $216 \mathrm{~K}$ are of particular interest. Examination of the diffraction pattern at $215 \mathrm{~K}$ showed pairs of reflections and, although the separation of the reflections was quite small, they are an indicator of twinning. However, at $216 \mathrm{~K}$ there are virtually no pairs of reflections; instead they are seen merged and take the form of smeared ellipses rather than separate discrete isotropic spots. Refinement of the orthorhombic model gave a similar result to that of the monoclinic model, and it is possible that there was a combination of both monoclinic and orthorhombic unit cells coexisting in equilibrium at the same time.

3.5.2. Structures at 217,218 and $219 \mathrm{~K}$. At these temperatures the balance begins to tip towards the orthorhombic crystal system. The first major observation at $217 \mathrm{~K}$ is that the non-merohedrally twinned crystal system is no longer an appropriate model for the data. Although GEMINI was able to determine two orientation matrices, refinement of the structure was poor, giving very high values of $R$ and $w R(0.133$ and 0.278 , respectively). The refined twin fraction had a very high uncertainty, thus making the parameter (and therefore the twinning) meaningless. As a result the non-merohedrally twinned monoclinic model was quickly discarded. A pure (i.e. untwinned) monoclinic model was tried, giving a slightly better result; however, both $A D D S Y M$ and ROTAX suggested that this model was no longer appropriate. Although the unconstrained $\beta$ angle is still almost a degree away from $90^{\circ}$, at $217 \mathrm{~K}$ the orthorhombic model gives the most satisfactory refinement result and we can say that the orthorhombic model is, on balance, the better way to describe the data. At 218 and $219 \mathrm{~K}$ the refinement results for the orthorhombic system become increasingly more favourable, and we now are more-or-less able to disregard the monoclinic crystal system as a reliable way of describing the structure; rather than being merely 'better described' as orthorhombic they are now clearly orthorhombic - a subtle but important difference.

\section{Conclusions}

The two previously reported crystal structures of barbituric acid dihydrate in space group Pnma only hold true at temperatures above $220 \mathrm{~K}$. Below $200 \mathrm{~K}$ the crystal structure is better described as non-merohedrally twinned monoclinic in space group $P 2_{1} / n$, and between 200 and $220 \mathrm{~K}$ the crystal structure undergoes a phase transition from monoclinic to orthorhombic. The phase transition is not particularly sharp; whilst the point at which the majority of the diffraction pattern changes from monoclinic to orthorhombic is probably around 216-217 K, the full transition appears to take place over a rather wider temperature range. The transition is reversible and the crystal suffers no physical effects as a result of either the temperatures used or the transition itself.

In the monoclinic structure the magnitude of the $\beta$ angle is seen to vary with temperature. The angle approaches $90^{\circ}$ as the temperature approaches the phase transition. There are no other significant changes in unit-cell dimensions and the observed increase in unit-cell volume is insignificant.

The structural differences in changing from the orthorhombic to monoclinic phase are most clearly seen by looking at the displacement of the $\mathrm{Csp}^{3}$ atom of the barbituric acid ring and the significant movement of the two water molecules away from coplanarity with the barbituric acid, as presented in Fig. 5. The orthorhombic phase features all atoms (with the exception of the $\mathrm{CH}_{2} \mathrm{H}$ atoms) lying on the mirror plane imposed by the space group, although in the monoclinic phase this is no longer a symmetry requirement and the molecules have the freedom to distort and shift. The hydrogen-bonding motif of both the orthorhombic and monoclinic phases is the same; however, the physical arrangement of the molecules is different, and this difference is best seen by viewing and comparing $c$-axis projections of the orthorhombic and monoclinic phases. 
We thank Dr Neil Brooks and Dr Ross Harrington, Newcastle University, for advice related to twinning and for experimental assistance, the EPSRC for financial support, and Professor Sally Price, University College London, for helpful discussions and pre-publication results relating to polymorph prediction for barbituric acid.

\section{References}

Al-Karaghouli, A. R., Abdul-Wahab, B., Ajaj, E. \& Al-Asaff, S. (1977). Acta Cryst. B33, 1655-1660.

American Chemical Society (2004). SciFinder Scholar Version 2004.2. http://www.cas.org/SCIFINDER/SCHOLAR/.

Brandenburg, K \& Putz, H. (2004). DIAMOND. Version 3. University of Bonn, Germany.

Bolton, W. (1963). Acta Cryst. 16, 166-173.

Bruker (2001). GEMINI, SMART and SAINT. Bruker AXS Inc., Madison, Wisconsin, USA.

Bruno, I. J., Cole, J. C., Edgington, P. R., Kessler, M., Macrae, C. F., McCabe, P., Pearson, J. \& Taylor, R. (2002). Acta Cryst. B58, 389397.

Caillet, J. \& Claverie, P. (1980). Acta Cryst. B36, 2642-2645.

Cleverley, B. \& Williams, P. P. (1959). Tetrahedron, 7, 277-288.

Cooper, R. I., Gould, R. O., Parsons, S. \& Watkin, D. J. (2002). J. Appl. Cryst. 35, 168-174.

Cosier, J. \& Glazer, A. M. (1986). J. Appl. Cryst. 19, 105-107.

Craven, B. M., Fox, R. O. \& Weber, H.-P. (1982). Acta Cryst. B38, 1942-1952.

Craven, B. M. \& Vizzini, E. M. (1969). Acta Cryst. B25, 1993-2009.
Craven, B. M. \& Vizzini, E. M. (1971). Acta Cryst. B27, 19171924.

Craven, B. M., Vizzini, E. M. \& Rodrigues, M. M. (1969). Acta Cryst. B25, 1978-1993.

Jeffrey, G. A., Ghose, S. \& Warwicker, J. O. (1961). Acta Cryst. 14, 881-887.

Lewis, T. C., Tocher, D. A. \& Price, S. L. (2004). Cryst. Growth Des. 4, 979-987.

Lewis, T. C., Tocher, D. A. \& Price, S. L. (2005). Cryst. Growth Des. 5, 983-993.

McMullan, R. K., Craven, B. M. \& Fox, R. O. (1978). Acta Cryst. B34, 3719-3722.

Nichol, G. S. \& Clegg, W. (2005a). Acta Cryst. C61, o297-o299.

Nichol, G. S. \& Clegg, W. (2005b). Acta Cryst. E61, o1004o1006.

Pandey, D. (2005). Acta Cryst. A61, 1-2.

Platteau, C., Lefebvre, J., Hemon, S., Baehtz, C., Danede, F. \& Prevost, D. (2005). Acta Cryst. B61, 80-88.

Sambyal, V. S., Goswami, K. N. \& Kahjuria, R. K. (1995). Cryst. Res. Technol. 30, 817-823.

Sheldrick, G. M. (2001). SHELXTL. Version 6. Bruker AXS Inc., Madison, Wisconsin, USA.

Sheldrick, G. M. (2002). TWINABS. University of Göttingen, Germany.

Sheldrick, G. M. (2003). SADABS. University of Göttingen, Germany.

Spek, A. L. (2003). J. Appl. Cryst. 36, 7-13.

Tomaszewski, P. (1992). Phase Transit. 38, 127-220.

Williams, P. P. (1973). Acta Cryst. B29, 1572-1579.

Williams, P. P. (1974). Acta Cryst. B30, 12-17. 